\title{
Perfil epidemiológico de óbitos por Covid-19 em Vitória da Conquista, Bahia Brasil
}

\author{
Epidemiological profile of deaths by Covid-19 in Vitória da Conquista, Bahia, Brazil \\ Perfil epidemiológico de las muertes por Covid-19 en Vitória da Conquista, Bahia, Brasil
}

Recebido: 24/11/2021 | Revisado: 01/12/2021 | Aceito: 07/12/2021 | Publicado: 16/12/2021

\author{
Carolina Neves Paixão \\ ORCID: https://orcid.org/0000-0002-7420-4685 \\ Faculdade Independente do Nordeste, Brasil \\ E-mail: Carolnevespx21@gmailcom \\ Jaina Esther de Mangueira Moreira \\ ORCID: https://orcid.org/0000-0003-0066-4002 \\ Faculdade Independente do Nordeste, Brasil \\ E-mail: esther_jaina@hotmail.com \\ Pablo Maciel Brasil Moreira \\ ORCID: https://orcid.org/0000-0002-0197-2894 \\ Prefeitura Municipal de Vitória da Conquista, Brasil \\ E-mail: pablomaciel.farmacia@gmail.com \\ Antonio Carlos Ricardo Braga Junior \\ ORCID: https://orcid.org/0000-0001-5870-4527 \\ Universidade Federal da Bahia, Brasil \\ E-mail: braga.ufba@hotmail.com \\ Aline Teixeira Amorim \\ ORCID: https://orcid.org/0000-0003-2597-8665 \\ Faculdade Independente do Nordeste, Brasil \\ E-mail: aline.amorim2011@ hotmail.com
}

\begin{abstract}
Resumo
Os coronavírus são vírus de RNA simples, positivo e não segmentado, além de possuírem um dos maiores genomas entre vírus de RNA. As discussões em torno destes vírus ressurgiram com o aparecimento de um novo coronavírus, o SARS-CoV-2, na cidade de Wuhan, China, no fim de 2019. Em março de 2020, a Organização Mundial da Saúde decretou o surto como pandemia, e o novo vírus chamou atenção por sua alta transmissibilidade, características intrínsecas e impacto social. Logo, o presente estudo tem por objetivo realizar o delineamento das características epidemiológicas que envolvem a mortalidade por SARS-CoV-2 no município de Vitória da Conquista, Bahia, Brasil. Realizou-se um estudo transversal, descritivo com abordagem quantitativa. Foram analisados todos os casos de óbitos por Covid-19 ocorridos entre abril de 2020 a agosto de 2021. Como instrumento de coleta de dados foram utilizados prontuários eletrônicos integrados no Sistema de Monitoramento da Covid-19 do município. Durante o período avaliado, foram analisados 33.539 dados de pacientes, dos quais 610 evoluíram para óbito. O perfil de óbito por Covid-19 no município é formado em sua maioria por pacientes do sexo masculino, de idade igual ou superior a 60 anos. Os sintomas: saturação de oxigênio menor 94\%, dificuldade/alterações respiratória e cianose, aliado a presença de comorbidades correlacionaram-se como fatores para maiores chances de óbitos na população estudada. Fica evidenciada a necessidade do desenvolvimento de políticas públicas voltadas a prevenção e cuidados dos pacientes com potencial risco ao óbito.
\end{abstract}

Palavras-chave: Coronavírus; Mortalidade; Perfil epidemiológico.

\begin{abstract}
Coronaviruses are simple, positive, and unsegmented RNA viruses, in addition to having one of the largest genomes among RNA viruses. Discussions around these viruses resurfaced with the appearance of a new coronavirus, SARSCoV-2, in the city of Wuhan, China, in late 2019. In March 2020, the World Health Organization declared the outbreak a pandemic, and the new virus drew attention for its high transmissibility, intrinsic characteristics and social impact. Therefore, the present study aims to outline the epidemiological characteristics that involve mortality from SARS-CoV-2 in the municipality of Vitória da Conquista, Bahia, Brazil. A cross-sectional, descriptive study with a quantitative approach was carried out. All cases of deaths from Covid-19 that occurred between April 2020 and August 2021 were analyzed. As a data collection instrument, electronic medical records integrated in the Covid-19 Monitoring System of the municipality were used. During the period evaluated, 33,539 data from patients were analyzed, of which 610 progressed to death. The profile of death by Covid-19 in the city is formed mostly by male patients, aged over 60 years. Symptoms: 94\% lower oxygen saturation, respiratory difficulties/changes and cyanosis, together with the presence of comorbidities, correlated as factors for greater chances of death in the population studied. The need for the development of public policies aimed at the prevention and care of patients with potential risk of death is evidenced.
\end{abstract}

Keywords: Coronaviruses; Mortality; Epidemiological profile. 


\section{Resumen}

Los coronavirus son virus de ARN simples, positivos y no segmentados, además de tener uno de los genomas más grandes entre los virus de ARN. Las discusiones en torno a estos virus resurgieron con la aparición de un nuevo coronavirus, el SARS-CoV-2, en la ciudad de Wuhan, China, a fines de 2019. En marzo de 2020, la Organización Mundial de la Salud declaró el brote como una pandemia y el nuevo virus se desató. atención por su alta transmisibilidad, características intrínsecas e impacto social, por lo que el presente estudio tiene como objetivo delinear las características epidemiológicas que involucran la mortalidad por SARS-CoV-2 en el municipio de Vitória da Conquista, Bahía, Brasil. Se realizó un estudio descriptivo transversal con abordaje cuantitativo. Se analizaron todos los casos de defunciones por Covid-19 ocurridos entre abril de 2020 y agosto de 2021. Como instrumento de recolección de datos se utilizó la historia clínica electrónica integrada en el Sistema de Monitoreo Covid-19 del municipio. Durante el período evaluado se analizaron 33.539 datos de pacientes, de los cuales 610 fallecieron. El perfil de muerte por Covid-19 en la ciudad está formado mayoritariamente por pacientes varones, mayores de 60 años. Síntomas: 94\% menor saturación de oxígeno, dificultades / alteraciones respiratorias y cianosis, junto con la presencia de comorbilidades, correlacionadas como factores de mayor probabilidad de muerte en la población estudiada. Se evidencia la necesidad del desarrollo de políticas públicas orientadas a la prevención y atención de pacientes en potencial riesgo de muerte.

Palabras clave: Coronavirus; Mortalidad; Perfil epidemiológico.

\section{Introdução}

A pandemia causada pelo novo coronavírus tem se mostrado um dos maiores desafios sanitários deste século (Who, 2021a). O surto ocasionado pelo vírus emergiu em Wuhan, cidade localizada na província de Hubei na China em meados de dezembro de 2019 (Zhu et al., 2020), relacionado com uma zoonose (Fleming et al., 2020).

A rápida disseminação dos casos da doença por vários países do mundo levou a Organização Mundial da Saúde (OMS) declarar a infecção por Covid-19 como pandemia no dia 11 de março do ano de 2020, considerando-a como emergência de saúde pública de importância internacional (Opas, 2020).

Nas últimas décadas houve outros dois tipos de coronavírus, no entanto, nenhum deles apresentou tamanha letalidade a nível global. Em 2003 houve um surto causado pelo SARS-CoV, com início em 2002, numa província de Guangdong, China. Segundo a OMS o episódio foi caracterizado como uma epidemia, alcançou 27 países, 8.096 números de casos e 774 mortes (Zhao et al., 2004). Dez anos depois do surgimento do primeiro SARS, em 2012, na Arábia Saudita surge a MERS-CoV (síndrome respiratória do Oriente Médio), que se alastrou por outros países. Em 2015 um novo surto de MERS-CoV ocorreu na Coreia do Sul, 1.728 pessoas foram infectadas (Pebmed, 2020). Após o surgimento do novo coronavírus, ao fim de fevereiro, os números eram espantosos, atingiu-se a marca de mais de 80.000 casos confirmados no mundo, espalhados por 51 países, mas sua maioria $(85,5 \%)$ eram habitantes da China (Steffens, 2020).

Os diversos coronavírus são vírus de RNA simples, positivo não segmentado e possuem um dos maiores genomas entre vírus de RNA. Pertencentes à família Coronaviridae, os coronavírus são agrupados em quatros gêneros: alfa-coronavírus, beta-coronavírus, gamma-coronavírus e delta- coronavírus. O novo coronavírus, segundo a taxonomia, é classificado como um beta-coronavírus, com capacidade de infectar humanos (Hartenian et al., 2020).

A transmissão da doença ocorre de diversos modos, em geral o contágio ocorre a partir da exposição de um indivíduo saudável a superfícies contaminadas (fômites) ou por meio do contato com indivíduos infectados, que podem se encontrar sintomáticos, pré-sintomáticos ou sem apresentação de sintomas (WHO, 2021b).

A doença apresenta espectro clínico amplo, podendo evoluir de um simples quadro gripal até uma pneumonia severa (Brasil, 2020a). Além disso, há ainda a possibilidade de evolução para quadro de sepse, falência renal aguda, insuficiência cardíaca e óbito (Chen et al., 2020). Ao entrar em contato com o organismo, o vírus, por meio da proteína S, se liga ao receptor da enzima conversora de angiotensina II (ACE2), presente em células epiteliais presentes em órgãos como pulmão, intestino, rim e vasos sanguíneos. Após a ligação vírus-célula hospedeira, as membranas se fundem e começa o processo de transcrição e replicação (Mojica-Crespo \& Morales-Crespo, 2020).

Algumas comorbidades estão associadas a uma forte expressão do receptor ACE-2 (Ejaz et al., 2020), visto que a 
expressão exacerbada destes receptores de enzima está potencialmente correlacionada com o aumento da suscetibilidade e progressão da doença (Pathangey et al., 2020). Neste sentido, compreende-se que a exposição ao novo coronavírus representa um risco maior em indivíduos comórbidos, com condições de saúde como hipertensão arterial (HA), diabetes mellitus (DM), doenças cardiovasculares (DCV), doenças respiratórias, doença renal crônica e câncer (Escoteguy et al., 2021). Duas ou mais dessas comorbidades podem coincidir em um mesmo indivíduo, representando um aumento significativo do risco de complicações pela doença (Brasil, 2020b).

Apesar da doença não demonstrar predileção por idade, evidências científicas apontam que pessoas idosas e com comorbidades tem maior probabilidade de desenvolver uma infecção grave pela Covid-19 assim como possuem maior probabilidade de evoluir para o óbito, quando comparados às demais faixas etárias, sendo assim, são considerados também como grupos de risco (Pathangey et al., 2020; Anghebem et al., 2020). Corroborando, um estudo realizado na Espanha apontou que dentre os 2.364 pacientes acometidos pela Covid-19 e que foram a óbito, um total de 1.117 pacientes possuíam comorbidades, percebeu-se ainda que a idade avançada também estabelecia relação com o agravo da doença (Vazquez et al., 2020).

A confirmação do primeiro caso no Brasil ocorreu no dia 26 fevereiro de 2020 e em março, houve a constatação da primeira morte por coronavírus, ambos ocorridos no estado de São Paulo (Porto et al., 2020). Em menos de três meses da notificação do primeiro caso de Covid-19 o país já contava mais de 100 mil pessoas infectadas e 7 mil óbitos, despertando interesse de órgãos internacionais que, começavam a projetar caos nos sistemas de saúde e aumento e/ou superlotação de leitos de unidade de terapia intensiva (Stein et al., 2020; Zimmermann, 2020). Até o dia 12 de setembro de 2021, cerca de 20 milhões casos e 586 mil óbitos já haviam sido notificados no país (Brasil, 2021). De acordo com o boletim epidemiológico divulgado pela Secretaria Municipal de Saúde (SMS) do município de Vitória da Conquista, até o dia 30 de agosto de 2021 às 17:00 horas, encontrava-se confirmados 34.729 casos de infecção por Covid-19 desde o início da pandemia, dos quais 610 evoluíram para óbito (PMVC, 2020).

Levando em consideração o cenário atual de índices altos de morbimortalidade dos grupos então considerados vulnerabilizados quando acometidos pela a infecção pelo novo coronavírus, é imprescindível entender o papel dos fatores de risco como modificadores do curso natural da doença ou como agravantes associados a pior evolução clínica e óbito, tendo em vista a necessidade de superar os desafios deste processo. Nesse sentido, a presente pesquisa objetiva delinear as características epidemiológicas que envolvem a mortalidade por Covid-19 no município de Vitória da Conquista, BA-Brasil.

\section{Metodologia}

Trata-se de um estudo transversal, descritivo com abordagem quantitativa. Os estudos de corte transversal descritivos são aqueles que observam a situação e características de determinada população ou fenômeno em um dado momento, possibilitam análises de correlação entre variáveis e desfechos que podem ou não estar associados (Aragão, 2011). O método quantitativo se caracteriza pelo emprego de métodos estatísticos, na coleta e análises dos dados, tem como finalidade medir relações entre as variáveis (Zanella, 2013).

O estudo foi realizado na cidade de Vitória da Conquista, município brasileiro do estado da Bahia. Segundo dados divulgados do último censo do Instituto Brasileiro de Geografia e Estatística (IBGE, 2020), o município apresenta uma área territorial de 3.254,186 km² a densidade demográfica de 91,41 (hab./km²) e uma população estimada de 343.643 habitantes, o que a classifica como a terceira maior cidade do estado, depois de Salvador e Feira de Santana. A população de estudo é composta por óbitos em decorrência da Covid-19 de indivíduos residentes no município, no período compreendido de 13 de abril de 2020 ( $1^{\circ}$ óbito) até o dia 30 de agosto de 2021. Foram excluídos da análise os óbitos por outras causas ou aqueles ainda em investigação. Como instrumento de coleta de dados, foram utilizados os prontuários eletrônicos integrados no Sistema de 
Monitoramento da Covid-19 do município.

Os participantes foram categorizados quanto às seguintes variáveis: faixa etária, sexo, comorbidades associadas e sintomatologia. Os dados coletados foram importados para o software Microsoft Excel® 2016, para organização e tabulação geral dos dados, subsequentemente importado para o programa SPSS Statistics (Versão 24), onde prosseguiu com a realização de análises estatísticas descritiva como frequências (absoluta e relativa), cálculos de medidas de tendência central como média, mediana e moda, medidas de dispersão (variância e desvio padrão).

As comparações das frequências por características foram analisadas por meio de estatísticas inferenciais, utilizando teste Qui-quadrado e análise do risco relativo, adotando um nível de significância de 5\%. O projeto foi aprovado pelo comitê de ética em pesquisa (CEP) em seres humanos sob parecer $n^{\circ} 5.068 .864$.

\section{Resultados}

Durante o período avaliado, foram extraídos do banco de dados 34.550 casos de Covid-19 e analisados 33.539 dados de pacientes, dos quais 610 evoluíram para óbito. Um total de 1.011 foram excluídos das análises por não conter informações sobre o real desfecho (óbito ou recuperado). No que tange a evolução dos óbitos, verifica-se que dois meses depois após a primeira notificação de morte por coronavírus em março de 2020, o número de mortes quadruplicou, em junho do mesmo ano já havia triplicado, chamando atenção para a rápida disseminação do vírus e sua capacidade de contágio. Desde então, os maiores registros de mortes foram no mês de março e maio de 2021 havendo depois um declínio no número de notificações (Figura 1).

Figura 1. Evolução mensal dos óbitos.

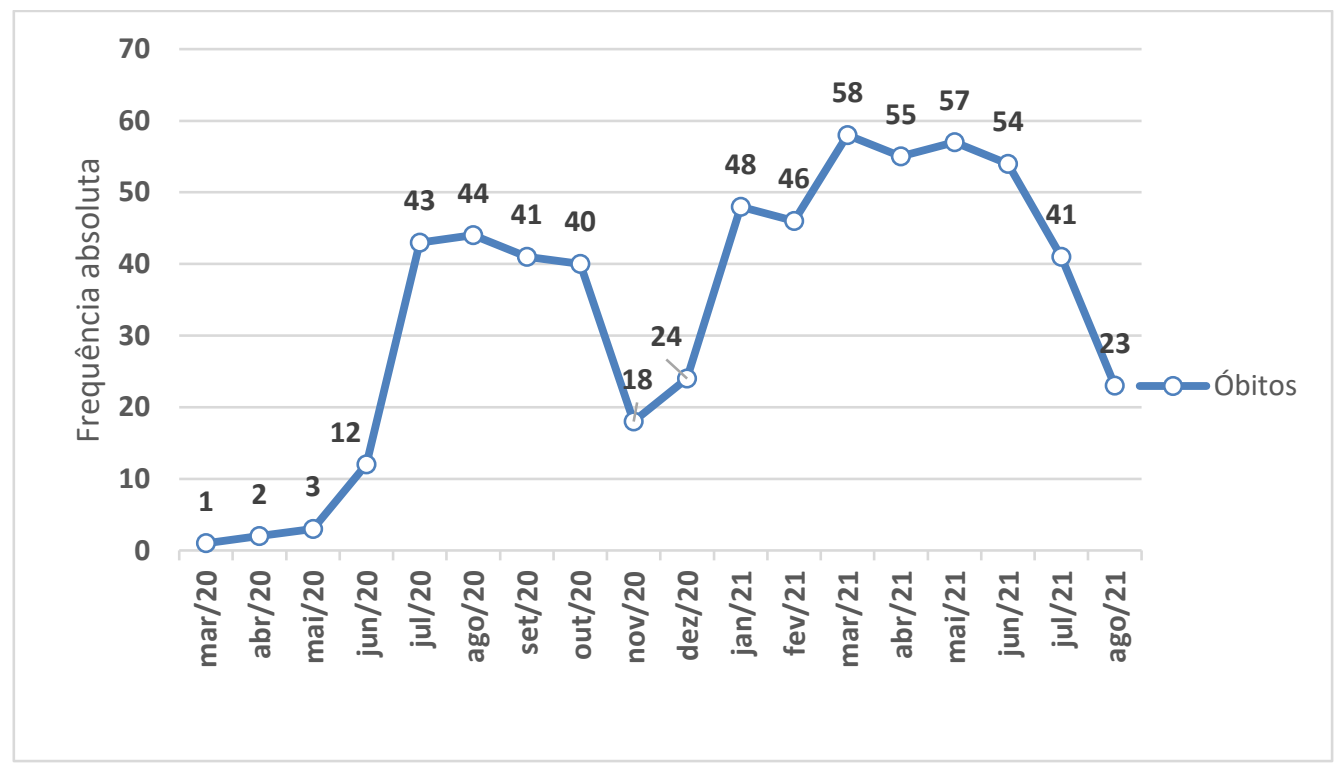

Fonte: Autores (2021).

De acordo com os dados apurados no período avaliado, constata-se uma maior incidência de óbitos por Covid-19 em pacientes do sexo masculino 58,9\% $(\mathrm{n}=359)$, com risco 1,8 vezes maior de evoluir para óbito quando comparado com o sexo feminino. Quanto a idade, paciente com idade igual ou superior a 60 anos apresentaram maior taxa de óbito (71,6\%), correlacionado aqueles com idade menor que 60 anos (28,4\%). Foram observados ainda que dentre os indivíduos acometidos pela doença com idade superior a 60 anos, apenas $12,7 \%(n=4.166)$ evoluíram para recuperação, em contrapartida, as faixas etárias menores de 60 anos somam 97,3\% dos casos de recuperação. Deste modo, infere-se que indivíduos com 60 anos ou mais, apresentam um risco de 17,4 vezes maior de evoluir para óbito quando comparado às demais faixas etárias (Tabela 1). 
Tabela 1. Distribuição de número de óbitos e não óbitos por faixa etária e sexo.

\begin{tabular}{|c|c|c|c|c|c|}
\hline \multirow[b]{2}{*}{ VARIÁVEIS } & \multicolumn{2}{|c|}{ ÓBITOS POR COVID } & \multirow[b]{2}{*}{$\begin{array}{l}\text { P(QUI-QUADRADO } \\
\text { DE PERSON })\end{array}$} & \multirow{2}{*}{$\begin{array}{c}\text { RAZÃO } \\
\text { DE } \\
\text { CHANCES }\end{array}$} & \multirow{2}{*}{$\begin{array}{c}\text { INTERVALO DF } \\
\text { CONFIANÇA } \\
(95 \%)\end{array}$} \\
\hline & $\begin{array}{c}\text { Sim } \\
\text { n }(\%)\end{array}$ & $\begin{array}{c}\text { Não } \\
\text { n (\%) }\end{array}$ & & & \\
\hline \multicolumn{6}{|l|}{ Sexo } \\
\hline Masculino & $359(58,9 \%)$ & $14.462(43,9 \%)$ & $<0,001$ & 1,826 & $1,552-2,149$ \\
\hline Feminino & $251(41,1 \%)$ & $18.467(56,1 \%)$ & & & \\
\hline \multicolumn{6}{|l|}{ Idade } \\
\hline$\geq 60$ & $437(71,6 \%)$ & $4.166(12,7 \%)$ & $<0,001$ & 17,440 & $14,581-20,859$ \\
\hline$<60$ & $173(28,4 \%)$ & $28.763(87,3 \%)$ & & & \\
\hline
\end{tabular}

Fonte: Autores (2021).

Em relação às comorbidades, as doenças cardiovasculares, incluindo HAS (35,9\%) e diabetes $(30,5 \%)$ foram as mais prevalentes na população estudada. Ao analisar a presença de comorbidades e sua correlação com risco de óbito, é possível inferir que estas representam significativa influência no desfecho da Covid-19. Os pacientes imunossuprimidos tinham risco de 10,97 vezes maior (I.C. $95 \%=5,717-21,076)$ de óbito. Um maior risco de óbito também está relacionado as seguintes comorbidades: doença cardiovascular, incluindo HAS, risco maior de 10,91 (I.C. 95\% = 9,184-12,977); doença neurológica crônica e neuromuscular, risco maior de 10,80 (I.C. 95\% =7,417-15,726); diabetes, risco maior de 8,87 (I.C. 95\% = 7,41510,623) e doença renal, risco maior de 8,17 (I.C. $95 \%=5,527-12,096)$ conforme Tabela 2.

Tabela 2. Correlação das comorbidades com os óbitos por Covid-19.

\begin{tabular}{|c|c|c|c|c|c|}
\hline \multirow[b]{2}{*}{ COMORBIDADES* } & \multicolumn{2}{|c|}{ ÓBITOS POR COVID } & \multirow[b]{2}{*}{$\begin{array}{l}\text { P(QUI-QUADRADO } \\
\text { DE PERSON })\end{array}$} & \multirow[b]{2}{*}{$\begin{array}{c}\text { RAZÃO } \\
\text { DE } \\
\text { CHANCES }\end{array}$} & \multirow{2}{*}{$\begin{array}{c}\text { INTERVALO } \\
\text { DE } \\
\text { CONFIANÇA } \\
(95 \%)\end{array}$} \\
\hline & $\begin{array}{c}\text { Sim } \\
\text { n }(\%)\end{array}$ & $\begin{array}{l}\text { Não } \\
\text { n (\%) }\end{array}$ & & & \\
\hline \multicolumn{6}{|l|}{$\begin{array}{l}\text { Doença cardiovascular, } \\
\text { incluindo HAS }\end{array}$} \\
\hline Sim & $219(35,9 \%)$ & $1.607(4,9 \%)$ & $<0,0001$ & 10,917 & $9,184-12,977$ \\
\hline Não & $391(64,1 \%)$ & $31.322(95,1 \%)$ & & & \\
\hline \multicolumn{6}{|l|}{ Diabetes } \\
\hline Sim & $186(30,5 \%)$ & $1.551(4,7 \%)$ & $<0,001$ & 8,875 & $7,415-10,623$ \\
\hline Não & $424(69,5 \%)$ & $31.378(95,3 \%)$ & & & \\
\hline \multicolumn{6}{|l|}{ Doença hepática } \\
\hline Sim & $10(1,6 \%)$ & $77(0,2 \%)$ & $<0,0001$ & 7,111 & $3,662-13,810$ \\
\hline Não & $600(98,4 \%)$ & $32.852(99,8 \%)$ & & & \\
\hline \multicolumn{6}{|l|}{$\begin{array}{l}\text { Doença neurológica } \\
\text { crônica e neuromuscular }\end{array}$} \\
\hline Sim & $34(5,6 \%)$ & $179(0,5 \%)$ & $<0,0001$ & 10,800 & $7,417-15,726$ \\
\hline Não & $576(94,4 \%)$ & $32.750(99,5 \%)$ & & & \\
\hline \multicolumn{6}{|l|}{ Imunossuprimido } \\
\hline Sim & $11(1,8 \%)$ & $55(0,2 \%)$ & $<0,0001$ & 10,976 & $5,717-21,076$ \\
\hline Não & $599(98,2 \%)$ & $32.87499,8 \%)$ & & & \\
\hline \multicolumn{6}{|l|}{ Doença renal } \\
\hline Sim & $30(4,9 \%)$ & $207(0,6 \%)$ & $<0,0001$ & 8,176 & $5,527-12,096$ \\
\hline Não & $580(95,1 \%)$ & $32.722(99,4 \%)$ & & & \\
\hline
\end{tabular}




\begin{tabular}{cccccc}
\hline Sim & $18(3,0 \%)$ & $166(0,5 \%)$ & $<0,0001$ & 6,001 & $3,665-9,826$ \\
Não & $592(97,0 \%)$ & $32.763(99,5 \%)$ & & & \\
Neoplasia & & & & & \\
Sim & $10(1,6 \%)$ & $88(0,3 \%)$ & $<0,0001$ & 6,220 & $3,218-12,022$ \\
$\quad$ Não & $600(98,4 \%)$ & $32.841(99,7 \%)$ & & & \\
Obesidade & & & & 1,773 & $1,235-2,545$ \\
Sim & $32(5,2 \%)$ & $997(3,0 \%)$ & $<0,0001$ & & \\
Não & $578(94,8 \%)$ & $31.932(97,0 \%)$ & & & \\
\hline
\end{tabular}

*Foram considerados somente os sintomas estatisticamente relevantes no teste Qui Quadrado, p < 0,05. Fonte: Autores (2021).

Quanto aos sintomas e o risco de óbito por Covid-19, foi possível observar que os pacientes que apresentaram saturação de oxigênio $\left(\mathrm{SpO}_{2}\right)$ menor que $94 \%$ tiveram risco de 44,33 vezes maior (I.C. $95 \%=36,759$ - 53,462) de ir a óbito em relação aos pacientes que apresentaram saturações maiores. Foram correlacionados também ao maior risco de óbito os sintomas: dificuldade de respirar progressiva, risco maior de 22,18 (I.C. $95 \%=18,395$ - 26,752); confusão mental, risco maior de 9,10 (I.C. 95\% = 4,288-19,326); cianose, risco de 7,39 (I.C. 95\% = 2,207-24,765) e respiração rápida ou lenta, risco maior de 2,51 (I.C. $95 \%=1,021-6,179)$. Os sintomas de congestão nasal, cefaleia, coriza e dor de garganta, se correlacionam com menores riscos de óbito, conforme dados apresentados na Tabela 3.

Tabela 3. Descrição dos sintomas apresentados pelos pacientes e a relação com o desfecho.

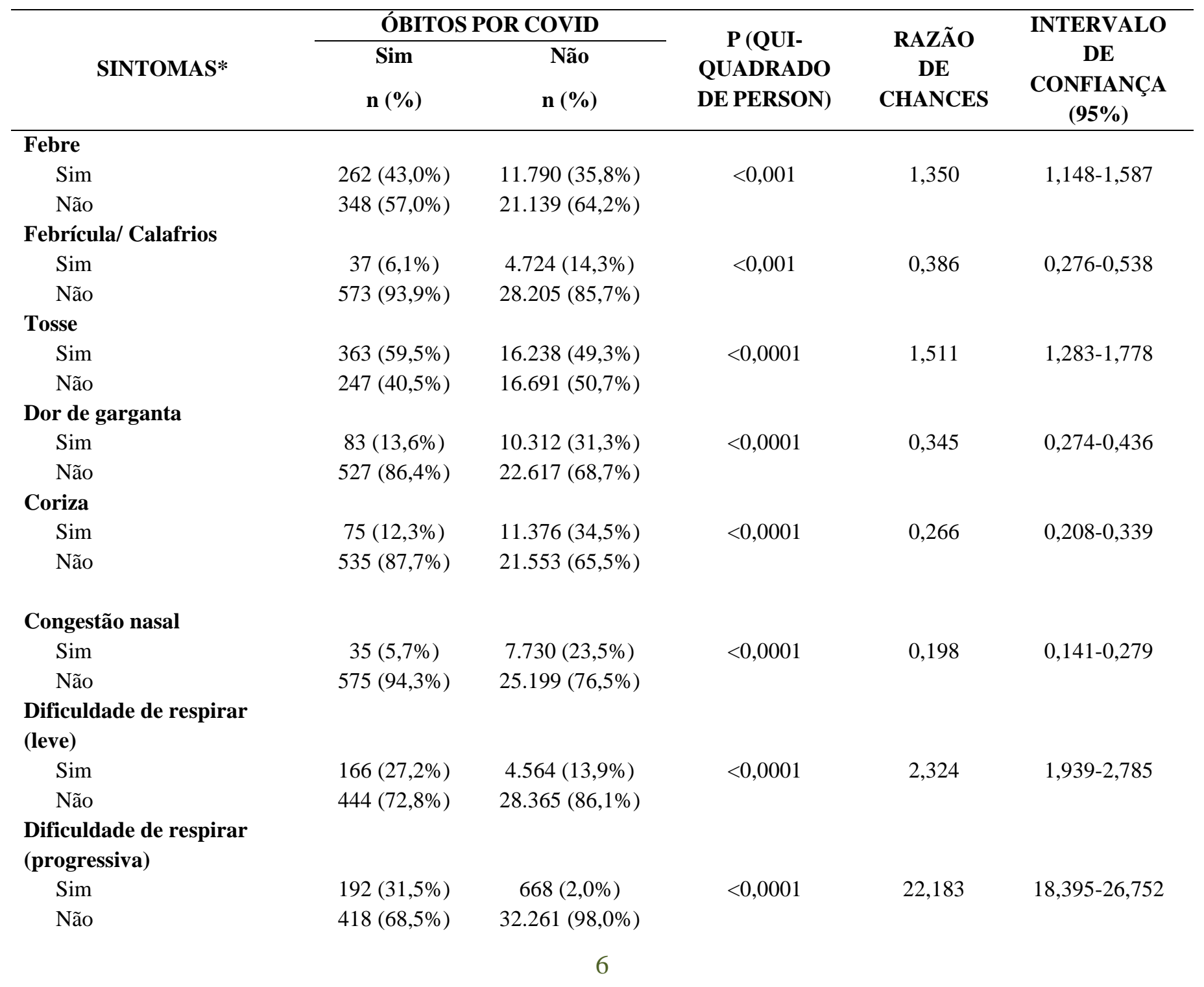




\begin{tabular}{|c|c|c|c|c|c|}
\hline \multicolumn{6}{|c|}{ Perda de paladar ou olfato } \\
\hline Sim & $62(10,2 \%)$ & $7.944(24,1 \%)$ & $<0,0001$ & 0,356 & $0,273-0,463$ \\
\hline Não & $548(89,8 \%)$ & $24.985(75,9 \%)$ & & & \\
\hline \multicolumn{6}{|c|}{$\mathrm{SpO}_{2}<94 \%$} \\
\hline Sim & $235(38,5 \%)$ & $459(1,4 \%)$ & $<0,0001$ & 44,331 & $36,759-53,462$ \\
\hline Não & $375(61,5 \%)$ & $32.470(98,6 \%)$ & & & \\
\hline \multicolumn{6}{|c|}{ Confusão mental } \\
\hline Sim & $8(1,3 \%)$ & $48(0,1 \%)$ & $<0,0001$ & 9,103 & $4,288-19,326$ \\
\hline Não & $602(98,7 \%)$ & $32.881(99,9 \%)$ & & & \\
\hline \multicolumn{6}{|l|}{ Cianose } \\
\hline Sim & $3(0,5 \%)$ & $22(0,1 \%)$ & $<0,0001$ & 7,393 & $2,207-24,765$ \\
\hline Não & $607(99,5 \%)$ & $32.907(99,9 \%)$ & & & \\
\hline \multicolumn{6}{|c|}{ Respiração rápida ou lenta } \\
\hline Sim & $5(0,8 \%)$ & $108(0,3 \%)$ & 0,038 & 2,512 & $1,021-6,179$ \\
\hline Não & $605(99,2 \%)$ & $32.821(99,7 \%)$ & & & \\
\hline \multicolumn{6}{|c|}{ Mialgia/artralgia } \\
\hline Sim & $126(20,7 \%)$ & $12.524(38,0 \%)$ & $<0,0001$ & 0,424 & $0,348-0,517$ \\
\hline Não & $484(79,3 \%)$ & $20.405(62,0 \%)$ & & & \\
\hline \multicolumn{6}{|l|}{ Cefaleia } \\
\hline Sim & $105(17,2 \%)$ & $16.253(49,4 \%)$ & $<0,0001$ & 0,213 & $0,173-0,264$ \\
\hline Não & $505(82,8 \%)$ & $16.676(50,6 \%)$ & & & \\
\hline \multicolumn{6}{|c|}{ Congestão conjuntival } \\
\hline Sim & $15(2,5 \%)$ & $1.806(5,5 \%)$ & $<0,001$ & 0,434 & $0,260-0,727$ \\
\hline Não & $55(97,5 \%)$ & $31.123(94,5 \%)$ & & & \\
\hline
\end{tabular}

*Foram considerados somente os sintomas estatisticamente relevantes no teste Qui Quadrado, p < 0,05. Fonte: Autores (2021).

\section{Discussão}

Boletins epidemiológicos emitidos pela prefeitura local indicam que Vitória da Conquista registrou 610 óbitos até o dia 30 de agosto de 2021. O município possui 343.643 habitantes, segundo o IBGE (2021), sendo o terceiro maior município do estado em população. Possui centros industriais, comércios, empresas e universidades que recebem munícipes vizinhos. Consequentemente, foi também o terceiro maior município em números de casos confirmados e em óbitos no estado. Na Bahia, a pandemia chegou a registrar 1.249.613 números de casos, evidenciando o alto índice de contágio da doença, diferente de todos os outros coronavírus já surgidos (Secretaria de Saúde do Estado da Bahia [SESAB], 2021).

Até a elaboração do presente estudo, o Brasil apresentou uma taxa de letalidade de 2,8\% (Brasil, 2021) semelhante a Bahia que teve 2,2\% (SESAB, 2021). Em Feira de Santana-BA, o índice de letalidade chegou a 1,9\% (Feira de Santana, 2021). A capital baiana, Salvador, apresentou uma letalidade de 3,22\% (Prefeitura Municipal de Salvador, 2021). Em Teresina-PI essa taxa chegou a alcançar 4,5\% (Silva et al., 2020). Já em Nova Xavantina-MT atingiu 57,1\% (Caló et al., 2020). Em comparação a outros estudos, nota-se que Vitória da Conquista apresentou um menor índice de letalidade: 1,8\% (PMVC, 2021).

Com relação a idade, este estudo analisou a prevalência de óbitos, com significância estatística, em pacientes com idade igual ou superior a 60 anos. Diversos estudos buscam entender a correlação entre o índice de óbitos por Covid-19 e o fator idade. Em análise dos fatores de multimorbidade e a população de risco para a doença, Nunes et al. (2020) observou que os 9.412 indivíduos com idade superior a 50 anos analisados apresentavam pelo menos uma comorbidade, o que justifica essa população compor o grupo de risco para Covid-19. Ademais, Escobar et al. (2020) complementa que além do perfil de comorbidade dos pacientes acima de 60 anos, no Brasil, as políticas públicas de integração do idoso a sociedade, a dificuldade do acesso e atenção em saúde, e ausência de programa de melhoria da 
qualidade de vida para maior idade, tornam essa população mais propensa aos prejuízos de situações de pandemia. Numa meta-análise, verificou-se que 39 estudos apontaram que pacientes com idade superior a 60 anos possuem uma eliminação prolongada do RNA viral (Cevik et al., 2021). O perfil de óbito por Covid-19 e a idade encontrado na presente análise corroboram com o perfil analisado em países europeus (WHO, 2020), no México (Bello-Chavolla et al., 2021) e na China (Wu et al, 2020), que demonstram maior índice e risco de óbito para pacientes com Covid-19 de idade superior a 60 anos.

Quanto ao sexo o presente estudo demonstrou maior índice de óbitos em pacientes do sexo masculino. Takahashi et al. (2020), analisando a resposta imunológica nos diferentes sexos, constatou que os pacientes do sexo masculino possuem um aumento de citocinas inflamatórias (IL-8, IL-18, CCL5), além disso, também foi verificado que durante o curso da doença os homens possuem uma menor ativação do número de células T. Em complemento, Mallah et al. (2021) traz que níveis elevados de alguns marcadores sanguíneos, entre ele citocinas inflamatórias, são associados a piores desfechos da Covid-19, uma vez que a associação com a tempestade de citocinas desencadeadas pela doença pode contribuir com o quadro hiper inflamatório. O sexo masculino demostra também uma eliminação de RNA viral mais prolongada, em relação ao sexo feminino (Cevik et al., 2021). Outros fatores da maior incidência masculina ao óbito refere-se as questões de isolamento social, onde o sexo masculino demonstra uma maior probabilidade de exposição ao risco e negligência da quarentena (Lima et al., 2020). Porto (2021) traz que a menor incidência de morte no sexo feminino pode estar relacionada ao estrogênio, que pode estimular melhor a imunidade além de responder melhor a vacinas. Fatores relacionados a essa incidência ainda é estudado e pode ser descrito melhor futuramente, mas estudos começam a ser desenvolvidos para evidenciar essas afirmações.

Quanto às comorbidades, pacientes imunossuprimidos, seguidos por doenças cardiovasculares, incluindo HAS, doenças neurológicas crônicas e neuromusculares, diabetes e doença renal apresentaram as maiores chances de óbitos, respectivamente. A presença de comorbidade em pacientes com Covid-19 já representa um fator de risco, e correlacionam-se com piores prognósticos (Souza et al., 2021). Ademais, a presença de uma comorbidade aumenta a chance de óbito em cerca de 9 vezes, em comparação aos pacientes sem comorbidades (Galvão \& Roncalli, 2021). Em análise sistemática de 11 estudos observou-se que a prevalência de doenças crônicas (doenças cardiovasculares, doenças pulmonares, hipertensão arterial, diabetes e doença renal) estão associados aos piores prognósticos em pacientes de Covid-19, bem como se associa a um alto risco de óbito (Arruda et al., 2021). Em Wuhan, um estudo revela que de 405 infectados, 79,1\% dos pacientes possuíam doenças crônicas (Zhan et al., 2020). Em Santa Catarina, 82,17\% dos pacientes que evoluíram para o óbito apresentavam comorbidades (Ramos, 2020), corroborando com os achados do presente estudo, onde mais de dois terços de pacientes infectados possuíam no mínimo uma comorbidade.

Quanto aos sintomas mais comuns nos casos avaliados, destacam-se os relacionados ao sistema respiratório, dificuldade de respirar leve e progressiva, diminuição da saturação de oxigênio e tosse. Somado à febre, estes foram os sintomas mais prevalentes, corroborando com estudos que descrevem o perfil clínico da doença (Pereira et al., 2020; Souza et al., 2021), Já os sintomas relacionados as maiores chances de óbitos por Covid-19 foram: saturação de oxigênio $\left(\mathrm{SpO}_{2}\right)<94 \%$, dificuldade de respirar leve e progressiva, respiração rápida e lenta e cianose. Estes sintomas são encontrados nos casos graves da doença e correlacionam-se como indicadores de piores prognósticos (Cespedes \& Souza, 2020). Os sintomas respiratórios podem cursar com desenvolvimento da síndrome da angústia respiratória aguda (SARA), um quadro pulmonar grave que evolui com a necessidade de ventilação mecânica assistida, o desenvolvimento dessa condição clínica contribui com o desenvolvimento de complicações e sequelas cognitivas, mentais e físicas, além da maior probabilidade do óbito (Campos et al., 2021; Souza et al., 2021).

Tais fatos podem ser explicados pela rota que o SARS-CoV-19 percorre no organismo humano, o vírus é transmitido através de gotículas aerossóis liberados por indivíduo infectado ou em contato com um objeto/superfície 
contaminada. As gotículas são os veículos responsáveis pela contaminação ao entrar em contato com a mucosa nasal ou orofaringe. O vírus utiliza a enzima conversora de angiotensina II (ACE2) para obter acesso às células alvo, presente nas células epiteliais situadas no pulmão, intestino, rim e vasos sanguíneos. Após a entrada o SARS-CoV-2 explora as células alveolares para realizar sua transcrição e replicação a fim de se espalhar por todo pulmão (Cespedes \& Souza, 2020).

Este estudo pode apresentar limitações, pois os dados trabalhados foram disponibilizados por boletins epidemiológicos, banco de dados secundários e veículos de informações de seus respectivos municípios, os quais pode haver informações incompletas. No entanto, as informações apresentadas neste trabalho possuem uma relevante aplicabilidade, uma vez que aborda o perfil epidemiológico de pacientes que vieram a óbito por COVID-19 no município de Vitória da Conquista. Assim, homens, de idades avançadas e com comorbidades apresentaram mais probabilidade de desfecho negativo da infecção.

\section{Conclusão}

Conforme análise dos resultados é possível inferir que o perfil de óbito por Covid-19 no município de Vitória da Conquista, Bahia, Brasil é formado em sua maioria por pacientes do sexo masculino, de idade igual ou superior a 60 anos. Ademais a presença de sintomas como $\mathrm{SpO}_{2}<94 \%$, dificuldade/alterações respiratória e cianose, bem como a presença de comorbidades correlacionaram-se como maiores chances de óbitos na população estudada. Os achados correlacionam-se com os dispostos em literatura, reafirmando o perfil clínico e os fatores de riscos associados aos óbitos por Covid-19.

Assim, neste contexto, fica evidenciada a necessidade do desenvolvimento de políticas públicas voltadas aos cuidados dos pacientes com potencial risco ao óbito. A compreensão do perfil epidemiológico de uma população e sua correlação com possíveis prognósticos podem auxiliar numa melhor análise situacional, adoção das medidas de controle, preparo na assistência médica, bem como na divulgação de orientações a população, contribuindo com possível redução de hospitalizações, óbitos e casos da doença.

O presente estudo alcança os objetivos propostos, espera-se com resultados encontrados contribuir nas discussões que dizem respeito ao perfil de óbito por Covid-19, bem como auxiliar na otimização de estratégias de combate a doença. Salientase ainda que esta pesquisa se mostra de grande relevância, trazendo dados importantes que podem servir de base para demais estudos que analisem os aspectos em torno da temática abordada. Ademais, se fazem necessários mais estudos que elucidem melhor como cada variável pode influenciar no desfecho da doença, bem como destaque as melhores ações a serem tomadas em cada caso.

\section{Referências}

Anghebem, M. I., Rego, F. G. de M., \& Picheth, G. (2020). COVID-19 e Diabetes: a relação entre duas pandemias distintas. RBAC, 52(2). doi:10.21877/24483877.20200001 .

Aragão, J. (2011). Introdução aos estudos quantitativos utilizados em pesquisas científicas. Revista Práxis, 3(6). https://doi.org/10.25119/praxis-3-6-566.

Arruda, D. É. G., Martins, D. D. S., Silva, I. F. M. da, \& Sousa, M. N. A. de. (2021). Prognóstico de pacientes com COVID-19 e doenças crônicas: Uma revisão sistemática. Comunicação Em Ciências Da Saúde, 31(03), 79-88.

Bello-Chavolla, O. Y., González-Díaz, A., Antonio-Villa, N. E., Fermín-Martínez, C. A., Márquez-Salinas, A., Vargas-Vázquez, A., Bahena-López, J. P., García-Peña, C., Aguilar-Salinas, C. A., \& Gutiérrez-Robledo, L. M. (2021). Unequal impact of structural health determinants and comorbidity on COVID-19 severity and lethality in older Mexican adults: Considerations beyond chronological aging. The Journals of Gerontology. Series A, Biological Sciences and Medical Sciences, 76(3), e52-e59.

BRASIL. (2020a). Protocolo de manejo clínico para o novo-coronavírus (2019-nCoV). Retrieved from Ministério da Saúde website: https://portalarquivos2.saude.gov.br/images/pdf/2020/fevereiro/11/protocolo-manejo-coronavirus.pdf

BRASIL. (2020b). Plano de contingência da Fiocruz diante da pandemia da doença pelo SARS-CoV-2 (Covid-19). Retrieved from Ministério da Saúde Fundação Oswaldo Cruz website: https://portal.fiocruz.br/sites/portal.fiocruz.br/files/documentos/plano_de_contingencia-covid19-fiocruzv2_2021-03-26.pdf

BRASIL. (2021). Coronavírus Brasil: Painel COVID-19. Retrieved from Ministério da Saúde website: https://covid.saude.gov.br/ 
Caló, R.S., Assis, J. M. V., Guenkka, T. M.., Pires, J. C. S., Andrade, A. C. S., Souza, R. A.G. (2020). Perfil epidemiológico dos óbitos por Coronavírus (COVID -19) em Mato Grosso. Saúde Coletiva (Barueri), 10(56), 3044-3055. https://doi.org/10.36489/saudecoletiva.2020v10i56p3044-3055

Campos, M. R., Schramm, J. M. de A., Emmerick, I. C. M., Rodrigues, J. M., Avelar, F. G. de, \& Pimentel, T. G. (2020). Carga de doença da COVID-19 e de suas complicações agudas e crônicas: reflexões sobre a mensuração (DALY) e perspectivas no Sistema Único de Saúde. Cadernos de Saude Publica, 36(11). https://doi.org/10.1590/0102-311x00148920

Cespedes, M. da S., \& Souza, J. C. R. P. de. (2020). Sars-CoV-2: A clinical update - II. Revista Da Associacao Medica Brasileira (1992), 66(4), 547-557.

Cevik, M., Tate, M., Lloyd, O., Maraolo, A. E., Schafers, J., \& Ho, A. (2021). SARS-CoV-2, SARS-CoV, and MERS-CoV viral load dynamics, duration of viral shedding, and infectiousness: a systematic review and meta-analysis. The Lancet. Microbe, 2(1), e13-e22.

Chen, T., Wu, D., Chen, H., Yan, W., Yang, D., Chen, G., ... Ning, Q. (2020). Clinical characteristics of 113 deceased patients with coronavirus disease 2019: retrospective study. BMJ (Clinical Research Ed.), 368, m1091.

Ejaz, H., Alsrhani, A., Zafar, A., Javed, H., Junaid, K., Abdalla, A. E., ... Younas, S. (2020). COVID-19 and comorbidities: Deleterious impact on infected patients. Journal of Infection and Public Health, 13(12), 1833-1839.

Escobar, A. L., Rodriguez, T. D. M., \& Monteiro, J. C. (2020). Lethality and characteristics of deaths due to COVID-19 in Rondônia: an observational study. Epidemiologia e Serviços de Saúde: Revista Do Sistema Único de Saúde Do Brasil, $30(1)$, e2020763.

Fleming, N., Sacks, L. J., Pham, C. T., Neoh, S. L., \& Ekinci, E. I. (2021). An overview of COVID-19 in people with diabetes: Pathophysiology and considerations in the inpatient setting. Diabetic Medicine: A Journal of the British Diabetic Association, $38(3)$, e14509.

Galvão, M. H. R., \& Roncalli, A. G. (2021). Fatores associados a maior risco de ocorrência de óbito por COVID-19: análise de sobrevivência com base em casos confirmados. Revista brasileira de epidemiologia [Brazilian journal of epidemiology], 23, e200106.

Hartenian, E., Nandakumar, D., Lari, A., Ly, M., Tucker, J. M., \& Glaunsinger, B. A. (2020). The molecular virology of coronaviruses. The Journal of Biological Chemistry, 295(37), 12910-12934.

IBGE. (2021). Cidades. Bahia, Vitória da Conquista. Retrieved from Instituto Brasileiro de Georgrafia e Estatisticas website: https://www.ibge.gov.br/cidades-e-estados/ba/vitoria-da-conquista.html

Lima, D. L. F., Dias, A. A., Rabelo, R. S., Cruz, I. D. da, Costa, S. C., Nigri, F. M. N., \& Neri, J. R. (2020). COVID-19 no estado do Ceará, Brasil: comportamentos e crenças na chegada da pandemia. Ciência \& Saúde Coletiva, 25(5), 1575-1586.

Mallah, S. I., Ghorab, O. K., Al-Salmi, S., Abdellatif, O. S., Tharmaratnam, T., Iskandar, M. A., Sefen, J. A. N., Sidhu, P., Atallah, B., El-Lababidi, R., \& AlQahtani, M. (2021). COVID-19: breaking down a global health crisis. Annals of Clinical Microbiology and Antimicrobials, $20(1), 35$.

Mojica-Crespo, R., \& Morales-Crespo, M. M. (2020). Pandemia COVID-19, la nueva emergencia sanitaria de preocupación internacional: una revisión. Semergen, 46 Suppl 1, 65-77.

Nunes, B. P., Souza, A. S. S. de, Nogueira, J., Andrade, F. B. de, Thumé, E., Teixeira, D. S. da C., Lima-Costa, M. F., Facchini, L. A., \& Batista, S. R. (2020). Multimorbidade e população em risco para COVID-19 grave no Estudo Longitudinal da Saúde dos Idosos Brasileiros. Cadernos de Saúde Publica, 36(12). https://doi.org/10.1590/0102-311x00129620

OPAS. (2020). Folha informativa - COVID-19. Retrieved from Organização Pan-Americana da Saúde website: https://www.paho.org/pt/news/11-3-2020who-characterizes-covid-19-pandemic

Pathangey, G., Fadadu, P. P., Hospodar, A. R., \& Abbas, A. E. (2021). Angiotensin-converting enzyme 2 and COVID-19: patients, comorbidities, and therapies. American Journal of Physiology. Lung Cellular and Molecular Physiology, 320(3), L301-L330.

Pebmed. (2020). MERS: a nova epidemia de coronavírus na Ásia. https://pebmed.com.br/mers-a-nova-epidemia-de-coronavirus-na-asia/

Pereira, M. D., Pereira, M. D., Costa, C. F. T., Santos, C. K. A. dos, \& Dantas, E. H. M. (2020). Aspectos epidemiológicos, clínicos e terapêuticos da COVID19. Journal of Health \& Biological Sciences, $8(1), 1$.

PMFS. (2021). Informe Epidemiológico Diário - COVID-19. Retrieved from Prefeitura Municipal de Feira de Santana website: feiradesantana.ba.gov.br.

PMVC. (2020). Boletim epidemiológico Coronavírus. Retrieved from Boletim epidemiológico Coronavírus website: https://www.pmvc.ba.gov.br/bol.etim$\% 20$ covid-19-sabado-sem-registro-de-obitos-e-de-novos-casos-em-vitoria-da-conquista

Porto, E. F., Domingues, A. L., Souza, A. C. de, Miranda, M. K. V., Froes, M. B. da C., \& Pasqualinoto, S. R. V. (2021). Mortalidade por Covid-19 no Brasil: perfil sociodemográfico das primeiras semanas. Research, Society and Development, 10(1), e34210111588.

Prefeitura Municipal de Salvador. (2021). Informe Salvador. Retrieved from Coronavirus website: http://informe.salvador.ba.gov.br/coronavirus/

Ramos, R. M. (2020). Análise do perfil epidemiológico dos óbitos por COVID-19 em Santa Catarina durante a pandemia de coronavírus até a 33a semana epidemiológica do ano de 2020. Retrieved from https://repositorio.ufsc.br/handle/123456789/218111

SESAB. (2021). COVID-19 na Bahia. Retrieved from Secretária Estadual de Saúde do Estado da Bahia website: https://infovis.sei.ba.gov.br/covid19/

Silva, T. M. da, Silva, L. E. A., Silva, A. R. da, Cavalcante, L. C. da S., Costa, S. de J., Silva, R. P. da, ... Santos, J. C. (2020). Perfil epidemiológico da morbimortalidade da Covid-19 no Estado do Piauí: uma atualização do cenário atual. Research, Society and Development, 9(8). doi:10.33448/rsd-v9i8.6091 
Souza, A. S. R., Amorim, M. M. R., Melo, A. S. de O., Delgado, A. M., Florêncio, A. C. M. C. da C., Oliveira, T. V. de, Lira, L. C. S., Sales, L. M. dos S., Souza, G. A., Melo, B. C. P. de, Morais, I., \& Katz, L. (2021). Errata: General aspects of the COVID-19 pandemic. Revista Brasileira de Saúde Materno Infantil, 21(suppl 2), 565-565.

Steffens, I. (2020). A hundred days into the coronavirus disease (COVID-19) pandemic. Euro Surveillance: Bulletin Europeen Sur Les Maladies Transmissibles [Euro Surveillance : European Communicable Disease Bulletin], 25(14). doi:10.2807/1560-7917.ES.2020.25.14.2000550

Stein, C., Cousin, E., Machado, Í. E., Felisbino-Mendes, M. S., Passos, V. M. de A., Sousa, T. M. de, ... Duncan, B. B. (2021). A pandemia da COVID-19 no Brasil: a série de projeções do Institute for Health Metrics and Evaluation e a evolução observada, maio a agosto de 2020. Epidemiologia e Servicos de Saude: Revista Do Sistema Unico de Saude Do Brasil, 30(1). doi:10.1590/s1679-49742021000100017

Takahashi, T., Ellingson, M. K., Wong, P., Israelow, B., Lucas, C., Klein, J., Silva, J., Mao, T., Oh, J. E., Tokuyama, M., Lu, P., Venkataraman, A., Park, A., Liu, F., Meir, A., Sun, J., Wang, E. Y., Casanovas-Massana, A., Wyllie, A. L., ... Iwasaki, A. (2020). Sex differences in immune responses that underlie COVID-19 disease outcomes. Nature, 588(7837), 315-320.

Vázquez-García, D., De-la-Rica-Escuín, M., Germán-Bes, C., \& Caballero-Navarro, A. L. (2020). Deaths in hospital emergency departments in Aragon: patient characteristics and association with comorbidity. Emergencias : Revista de La Sociedad Espanola de Medicina de Emergencias, 32(3), $162-168$.

WHO. (2021a). Coronavirus (COVID-19) Dashboard. Retrieved from World Health Organization website: https://covid19.who.int/. Acesso em: 20 maio 2021

WHO. (2020). COVID-19: WHO european region operational update epi weeks 31-32. World Health Organization. https://www.euro.who.int/_data/assets/pdf_file/0008/460196/COVID-19-operational-update-weeks-31-32-eng.pdf

WHO. (2021b). Transmission of SARS-CoV-2: implications for infection prevention precautions. Retrieved from World Health Organization website: https://www.who.int/news- room/commentaries/detail/transmission-of-sars-cov-2-implic ations-for-infection-prevention-precautions.

Wu, D., Wu, T., Liu, Q., \& Yang, Z. (2020). The SARS-CoV-2 outbreak: What we know. International Journal of Infectious Diseases: IJID: Official Publication of the International Society for Infectious Diseases, 94, 44-48.

Zanella, L. $\quad$ C. H., Metodologia (2013). de http://arquivos.eadadm.ufsc.br/EaDADM/UAB_2014_2/Modulo_1/Metodologia/material_didatico/Livro\%20texto\%20Metodologia\%20da\%20Pesquisa.pdf.

Zimmermann, I., Sanchez, M., Brant, J., \& Alves, D. (2020). Projeção de internações em terapia intensiva pela COVID-19 no Distrito Federal, Brasil: uma análise do impacto das medidas de distanciamento social. Epidemiologia e Serviços de Saúde: Revista Do Sistema Único de Saúde Do Brasil, 29(5). https://doi.org/10.1590/s1679-49742020000500022.

Zhan, T., Liu, M., Tang, Y., Han, Z., Cheng, X., Deng, J., ... Huang, X. (2020). Retrospective analysis of clinical characteristics of 405 patients with COVID19. The Journal of International Medical Research, 48(8), 300060520949039.

Zhao, Z., Li, H., Wu, X., Zhong, Y., Zhang, K., Zhang, Y.-P., ... Fu, Y.-X. (2004). Moderate mutation rate in the SARS coronavirus genome and its implications. BMC Evolutionary Biology, 4, 21.

Zhu, N., Zhang, D., Wang, W., Li, X., Yang, B., Song, J., ... Tan, W. (2020). A novel Coronavirus from patients with pneumonia in China, 2019. The New England Journal of Medicine, 382(8), 727-733. 